\title{
Effective Radiotherapy in Tumor Assisted by Ganoderma lucidum Polysaccharide-Conjugated Bismuth Sulfide Nanoparticles through Radiosensitization and Dendritic Cell Activation
}

Huan Yü, Yang Yang ${ }^{\dagger}$, Tianyan Jiang ${ }^{\dagger}$, Xihui Zhang ${ }^{\dagger}$, Yuhao Zhao ${ }^{\dagger}$, Guibin Pang ${ }^{\dagger}, \xi$, Yahui Feng", Shulei Zhangt,, , Fujun Wangt,, , Yong Wangt, Yangyun Wang ${ }^{\dagger}$ *, Leshuai W Zhang ${ }^{\dagger}$ *

* Corresponding authors:

Yangyun Wang, School of Radiation Medicine and Protection, State Key Laboratory of Radiation Medicine and Protection, School for Radiological and Interdisciplinary Sciences (RAD-X), Collaborative Innovation Center of Radiation Medicine of Jiangsu Higher Education Institutions, Soochow University, Suzhou, 215123, China

Email: yywang578@suda.edu.cn Phone: +8651265882942

Leshuai W Zhang, School of Radiation Medicine and Protection, State Key Laboratory of Radiation Medicine and Protection, School for Radiological and Interdisciplinary Sciences (RAD-X), Collaborative Innovation Center of Radiation Medicine of Jiangsu Higher Education Institutions, Soochow University, Suzhou, 215123, China

Email:zhangls@suda.edu.cn Phone:+8651265882942 


\section{Content of Supplementary Figures}

\begin{tabular}{cccc}
\hline \multirow{2}{*}{ Nanoparticles } & \multicolumn{3}{c}{ Surface charge $(\mathrm{mV})$} \\
\cline { 2 - 4 } & Water & PBS & DMEM \\
\hline BiNP & $-28.5 \pm 1.45$ & $-16.8 \pm 0.59$ & $-16.07 \pm 2.6$ \\
\multirow{2}{*}{ GLP-BiNP } & $-20.93 \pm 0.54$ & $-14.3 \pm 0.89$ & $-13 \pm 0.43$ \\
\hline
\end{tabular}

Supplementary Information Table S1. Surface charges of BiNP and GLP-BiNP. Values are presented as the mean \pm S.D. $(n=3)$ 

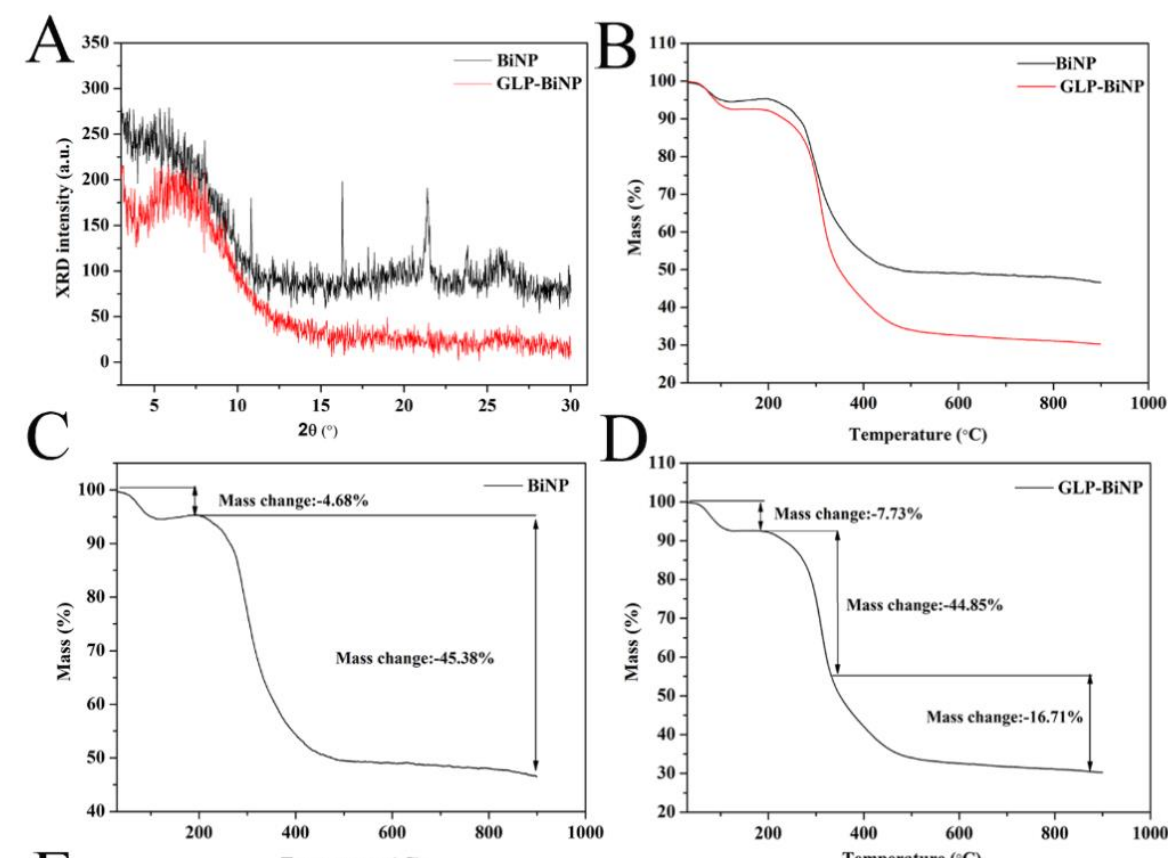

$\mathrm{E}$

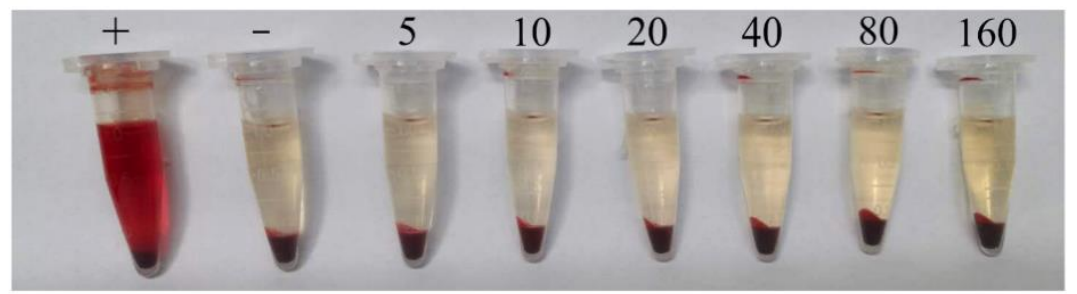

Figure S1. Other characterization data of GLP-BiNP. (A) X-ray diffraction of BiNP and GLP-BiNP. (B) Thermogravimetric analysis (TGA) of BiNP and GLP-BiNP with temperature increment from 30 to $900^{\circ} \mathrm{C}$ at a heating rate of $20^{\circ} \mathrm{C} / \mathrm{min}$ in a nitrogen atmosphere. Specifically, the TGA curves are marked with percentage of mass changes as a function of increasing temparature for BiNP (C) and GLP-BiNP (D). (E) Hemolytic behavior of red blood cells (RBCs) by the different concentrations GLP-BiNP, and $0.9 \%$ normal saline was set as negative control and the distilled water as the positive control. 


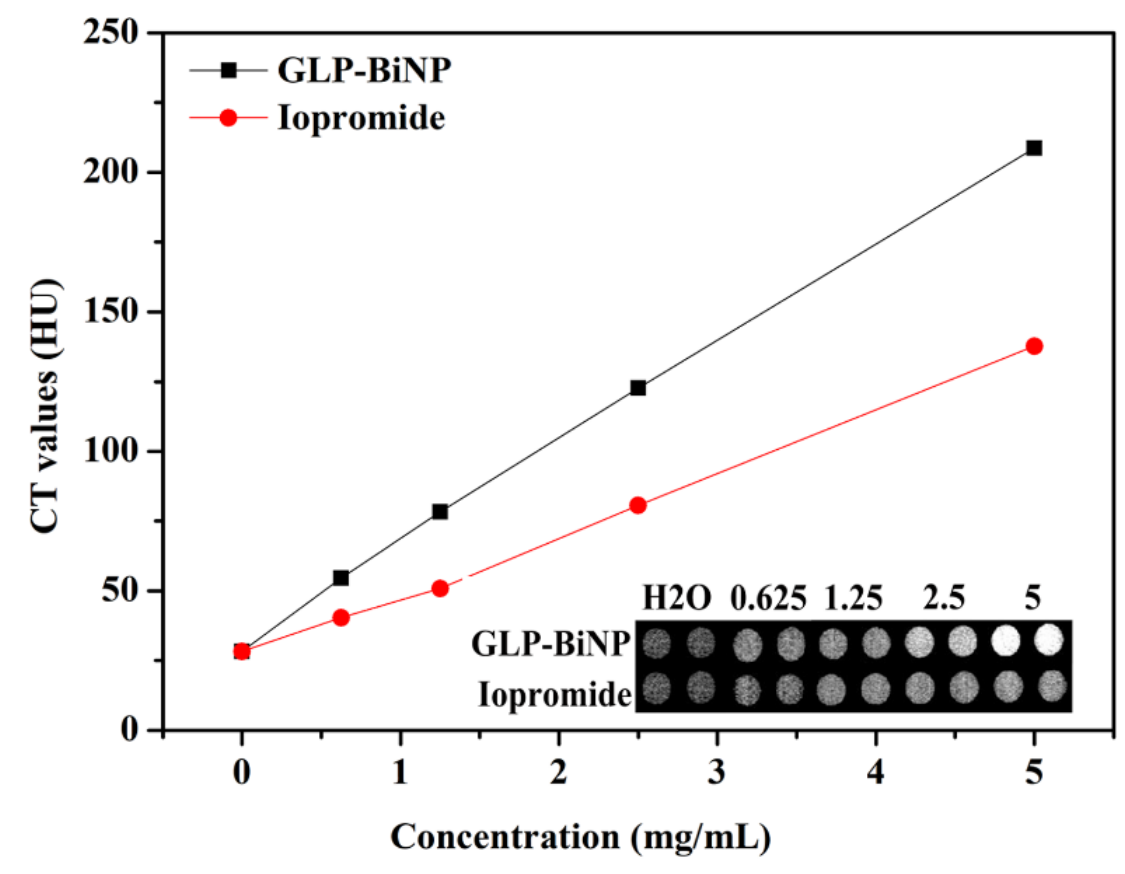

Figure S2. CT images and values of GLP-BiNP. CT imaging parameters were set at full angle with tube current at $615 \mu \mathrm{A}$ and voltage of $55 \mathrm{kV}$. (A) A photograph of as-prepared GLP-BiNP (2.5 $\mathrm{mg} / \mathrm{mL}$ ) in deionized water, suggesting the nanoparticle formation. (B) CT values of GLP-BiNP and the standardized iopromide at different concentrations. Inset: CT images of GLP-BiNP and iopromide solutions in deionized water. 


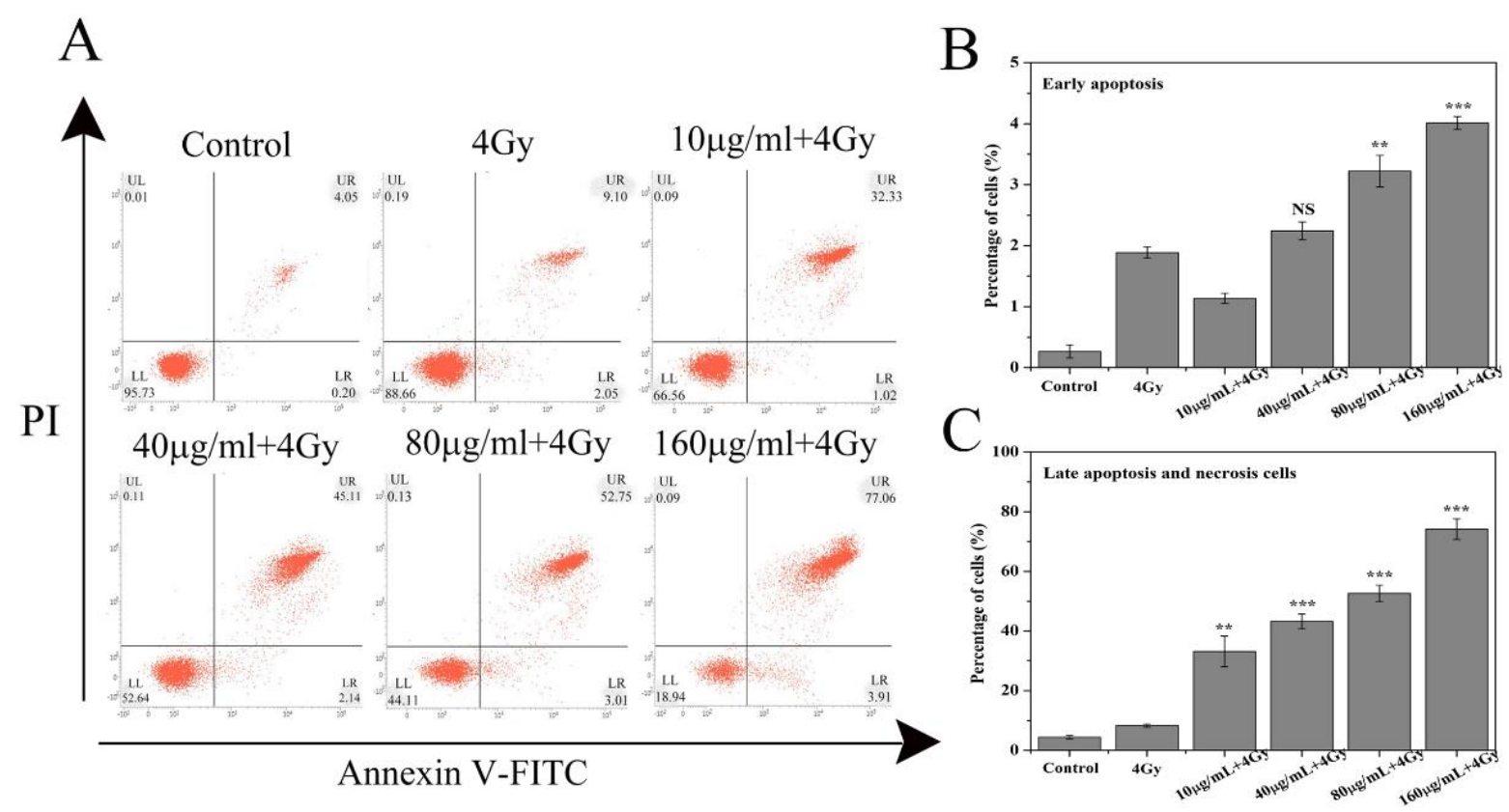

Figure S3. Cell apoptosis of 4T1 cells by radiation in the presence of GLP-BiNP. (A) 4 T1 cells were subjected to X-ray alone, or X-ray with different concentrations of GLP-BiNP. Cells were collected and the percentages of early and late apoptotic/necrotic cells were determined by flow cytometry. Percentage of early apoptosis (B) and late apoptosis/necrosis (C) under different treatments $(n=3)$. Statistical analysis was perfromed with one-way ANOVA (NS: Non-significant, $* * P<0.01$ and $* * * P<0.001$ indicate the differences between $\mathrm{X}$-ray treatment and other treatment samples). Error bars represent mean \pm S.D. 


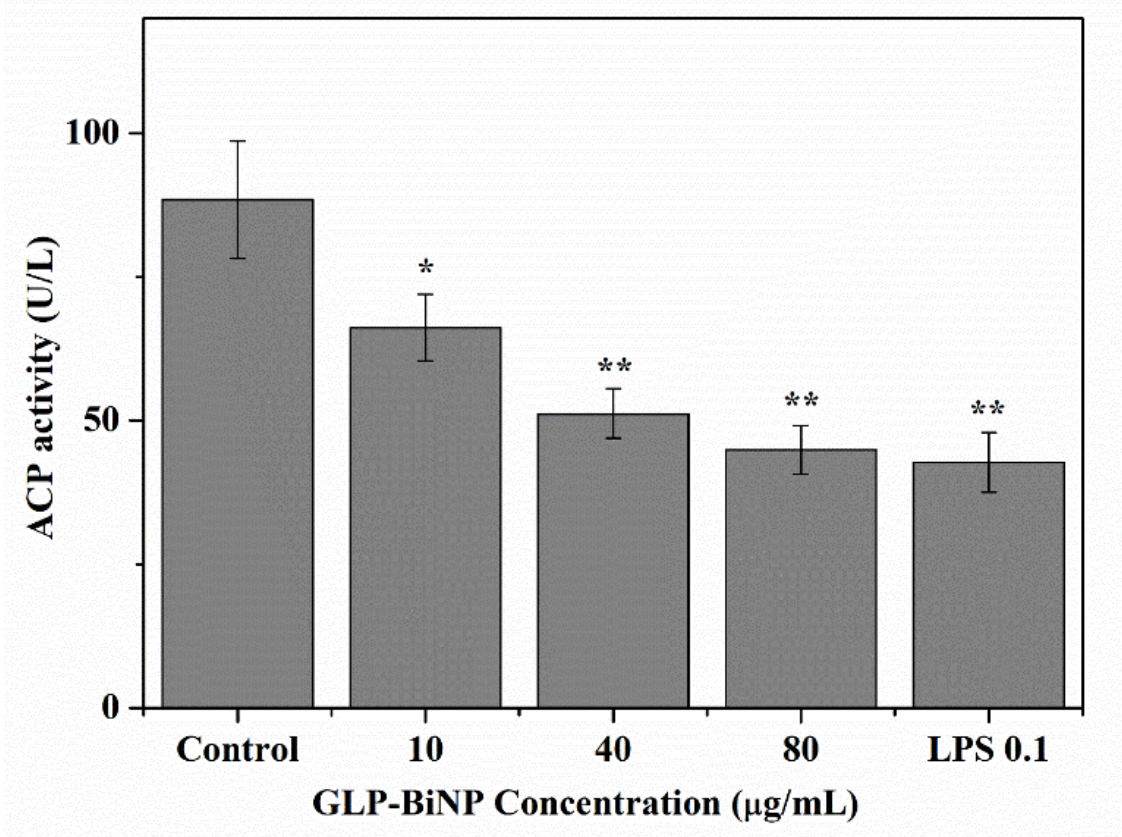

Figure S4. ACP activity of DC. DC were treated with 10,40 or $80 \mu \mathrm{g} / \mathrm{mL}$ of GLP-BiNP for $24 \mathrm{~h}$ and ACP activity was assayed. LPS at $0.1 \mu \mathrm{g} / \mathrm{mL}$ was set as the positive control. Statistical analysis was perfromed with one-way ANOVA $\left({ }^{*} P<0.05\right.$ and ${ }^{* *} P<0.01$ compared to control). Error bars represent mean \pm S.D. $(\mathrm{n}=6)$. 


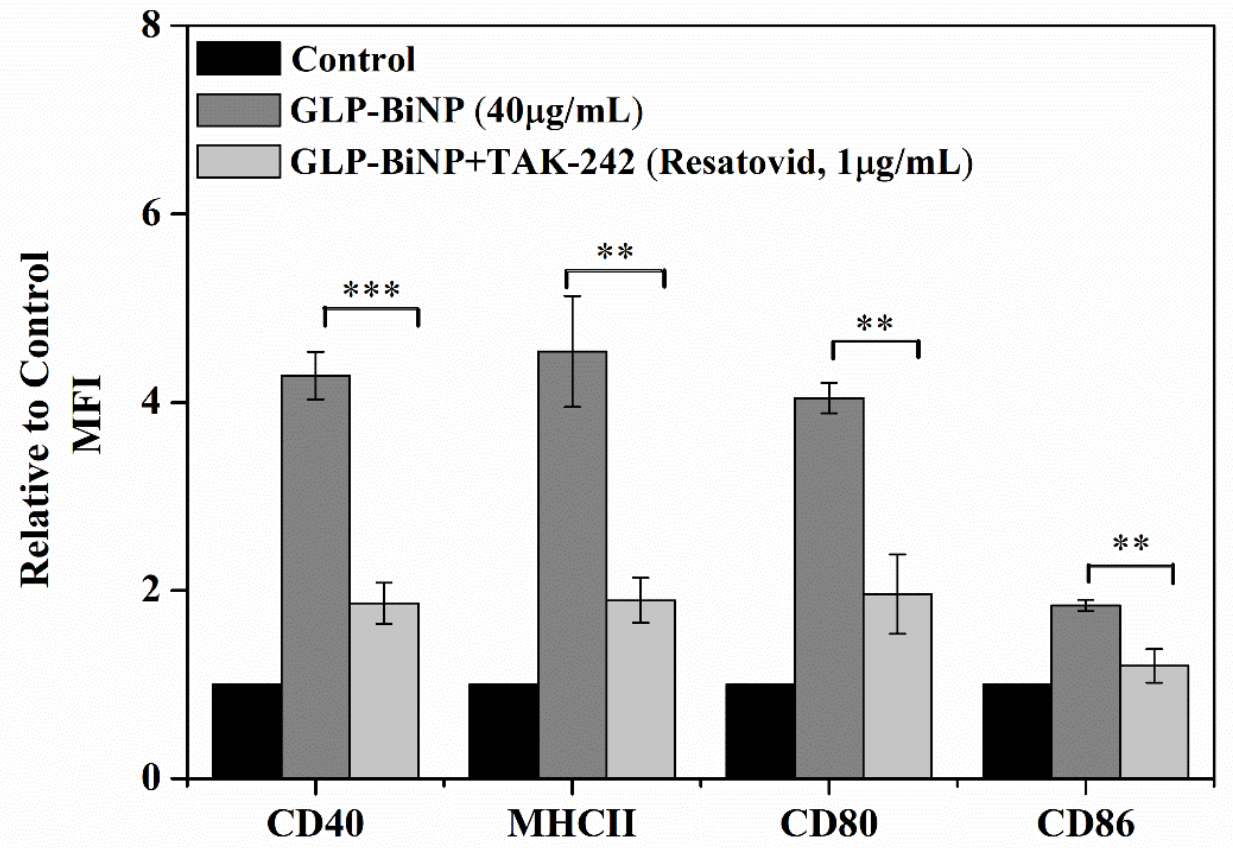

Figure S5. The effect of toll-like receptor-4 inhibitor on the protein expression of DC maturation markers. DC were treated with PBS, or GLP-BiNP with/without TAK-242, a toll-like receptor 4 inhibitor. Statistical analysis was perfromed with one-way ANOVA ( $* * P<0.01$ and $\left.{ }^{* * *} P<0.001\right)$. Error bars represent mean \pm S.D. MFI: Mean Fluorescence Intensity. 


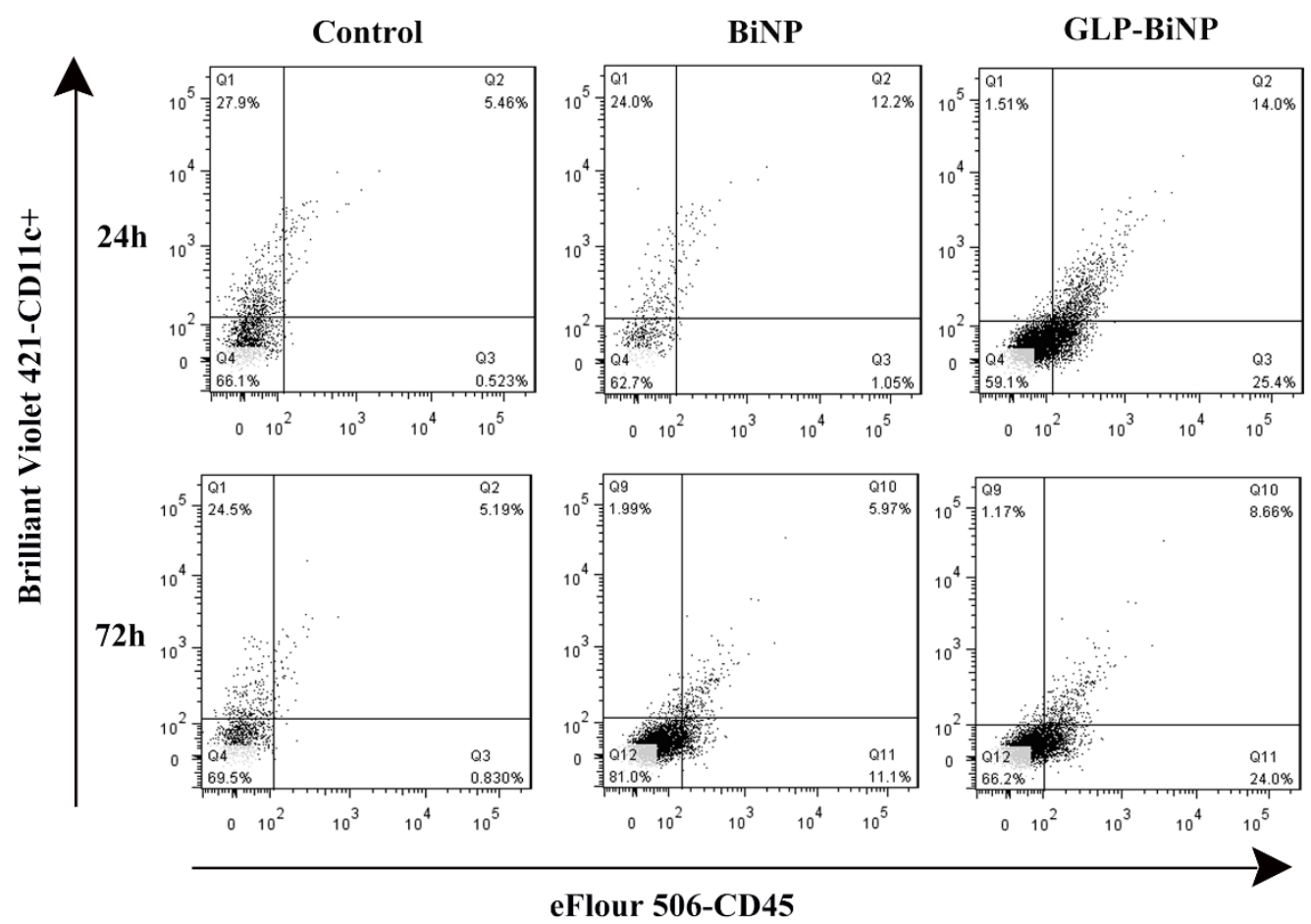

Figure S6. Scatter plots for intratumor DC in mice with different treatment. Tumor bearing mice were subjected to PBS, BiNP and GLP-BiNP. Tumor were collected $24 \mathrm{~h}$ and $72 \mathrm{~h}$ later and digested for DC analysis by flow cytometry, shown as the scatter plots. In $\mathrm{CD} 45^{+}$lymphocytes, $\mathrm{CD} 11 \mathrm{c}^{+} \mathrm{DC}$ were selected for percentage calculation in lymphocytes. 

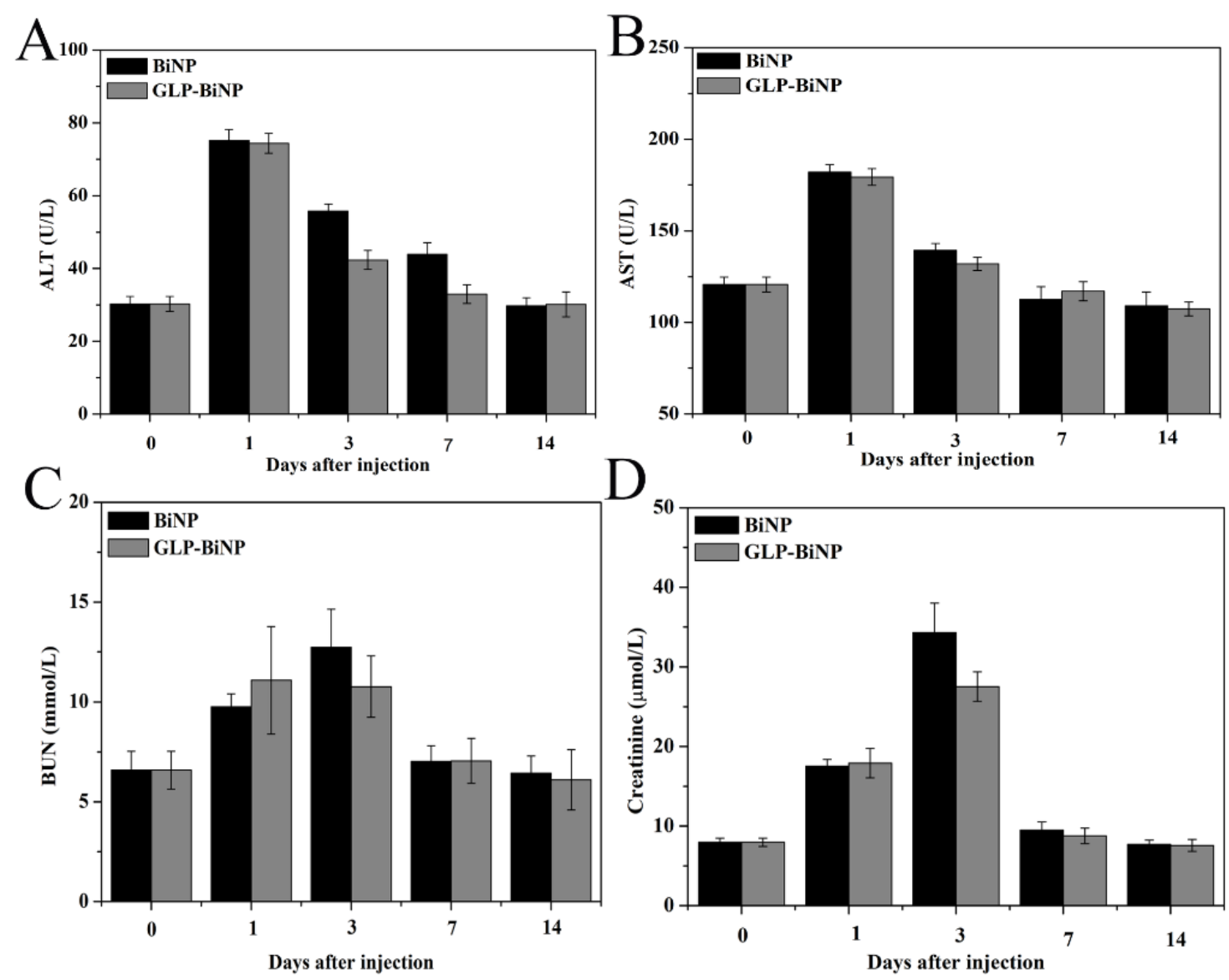

Figure S7. Blood biochemical tests of mice. Mice were intravenously injected with BiNP (black bars), GLP-BiNP (gray bars) and the serum were collected at day 1, 3, 7, and 14 for blood biochemical assays. (A) Alanine aminotransferase; (B) Aspartate aminotransferase; (C) Blood urea nitrogen; (D) Creatinine. Error bars represent mean \pm S.D. $(n=3)$. 


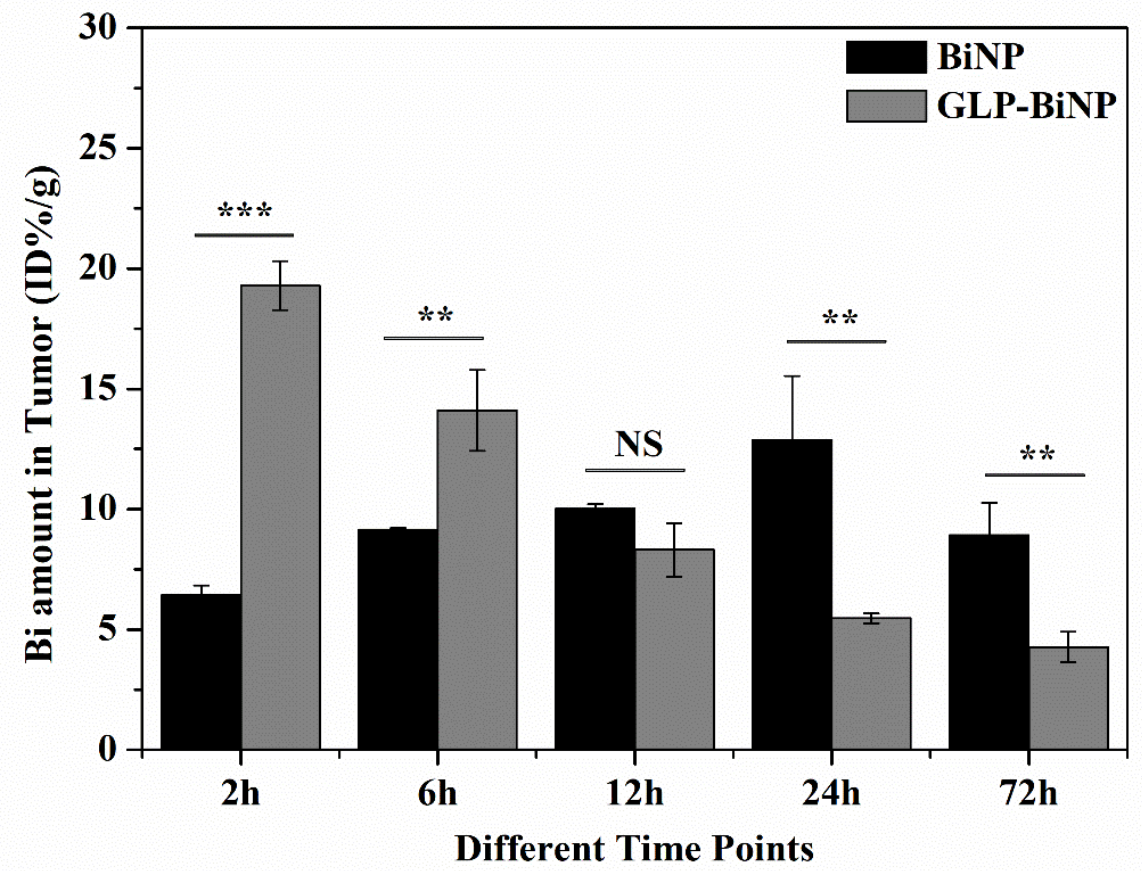

Figure S8. Bismuth element quantification for BiNP and GLP-BiNP in tumor tissues with different time points. $P$ values were calculated by one-way ANOVA (NS:Non-significant, $\left.{ }^{* *} P<0.01, * * * P<0.001\right)$. 


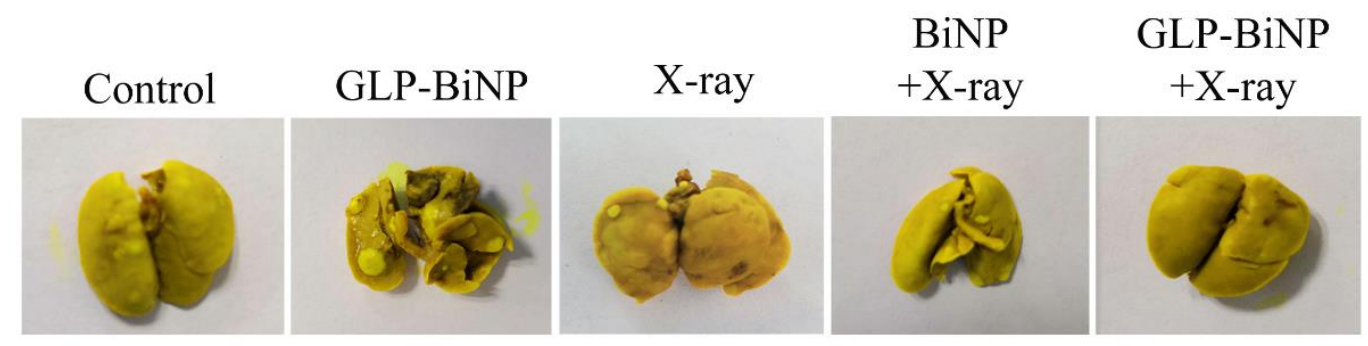

Figure S9. A photograph of the metastasis inhibition by the combination treatment. Fresh lungs collected on day 14 and fixed with Bouin's fluid. 


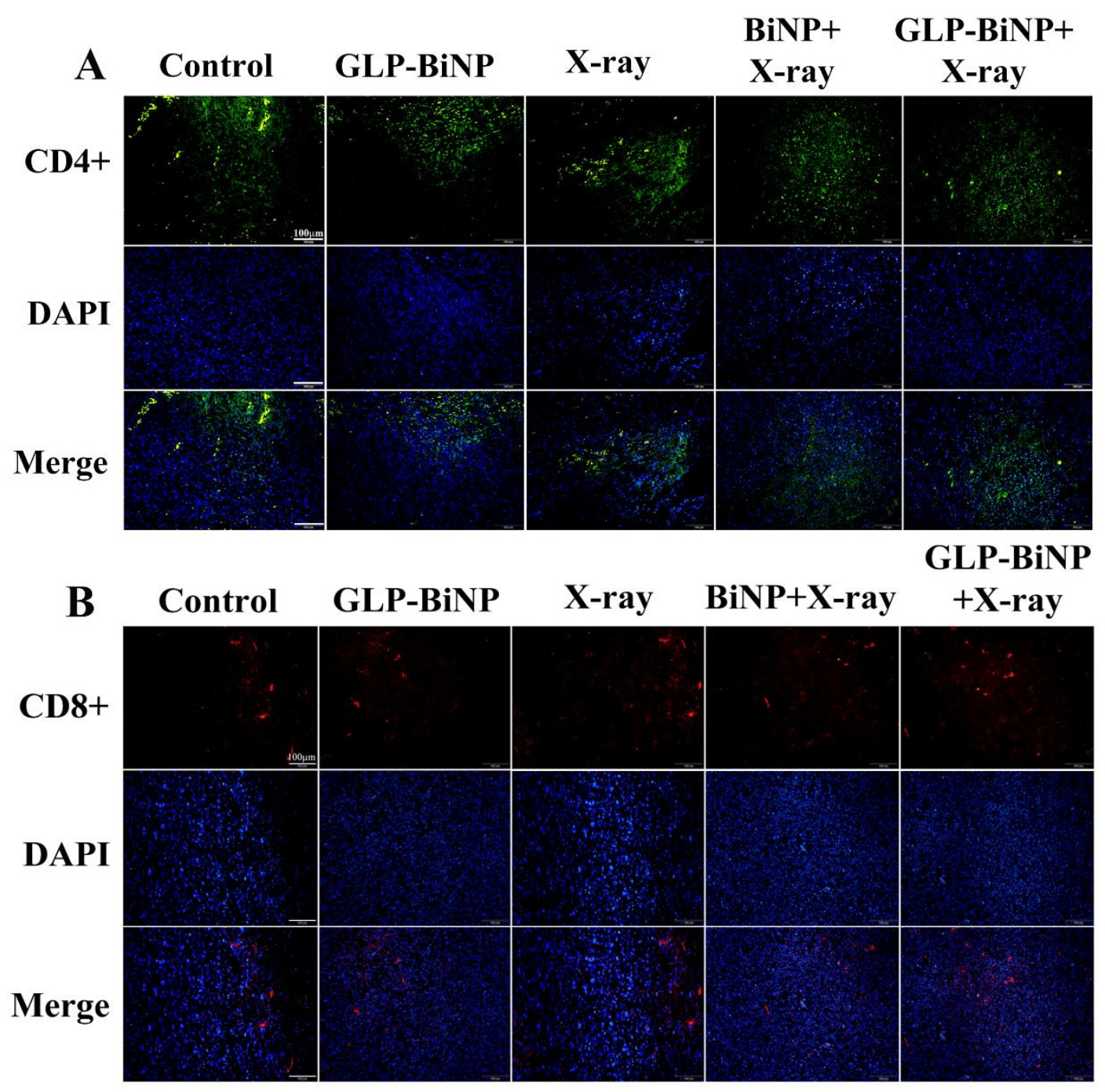

Figure S10. Tumor-infiltrating $\mathrm{CD}^{+} \mathrm{T}$ cells and $\mathrm{CD8}^{+} \mathrm{T}$ cells in the tumors. Tumors collected on day 14 were fixed, paraffin embedded and sectioned for immunofluorescence staining of CD4 (A) and CD8 (B). The scale bar represents $100 \mu \mathrm{m}$. 


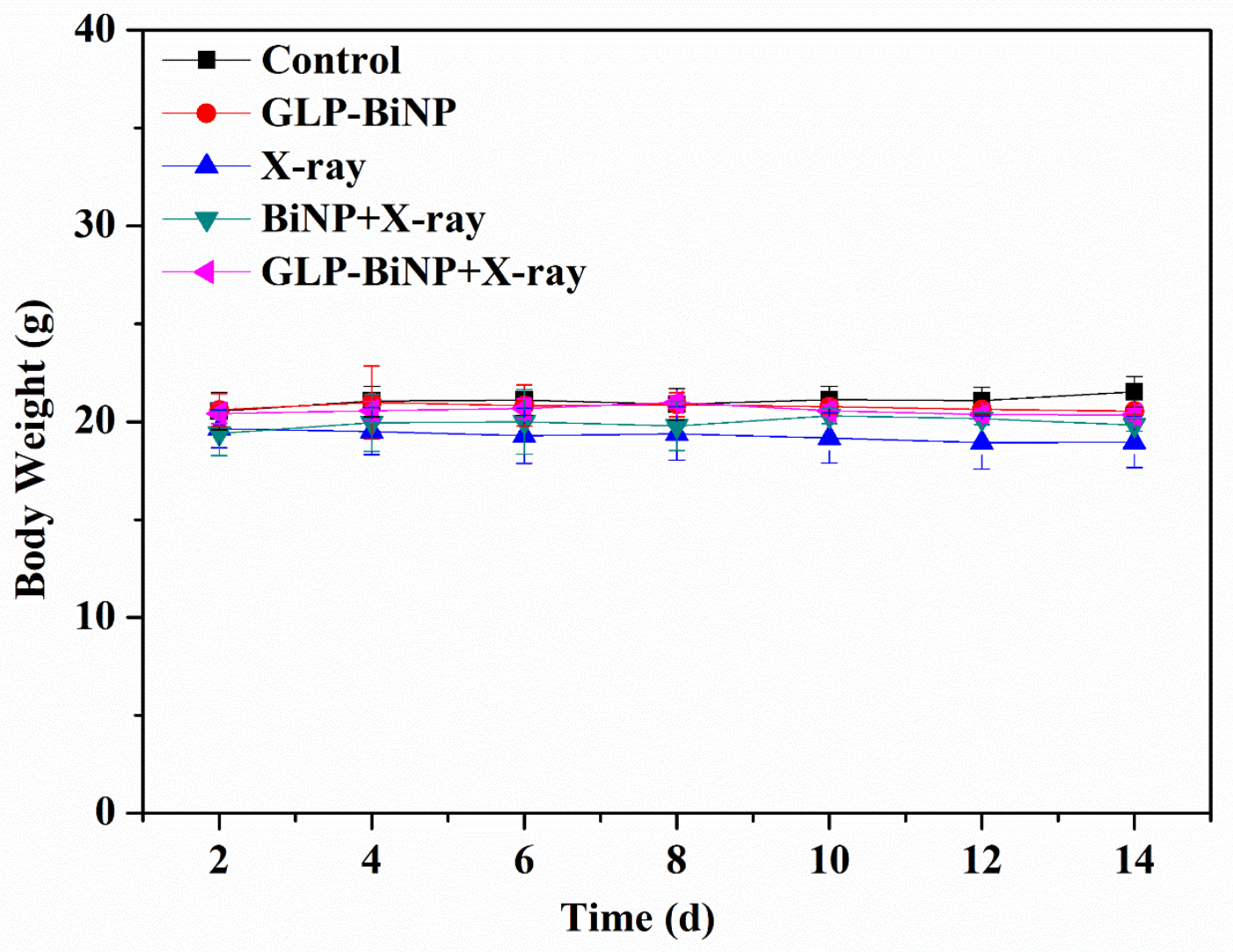

Figure S11. Body weights of mice bearing 4T1 tumors with time. Error bars represent mean \pm S.D. $(n=6)$ 


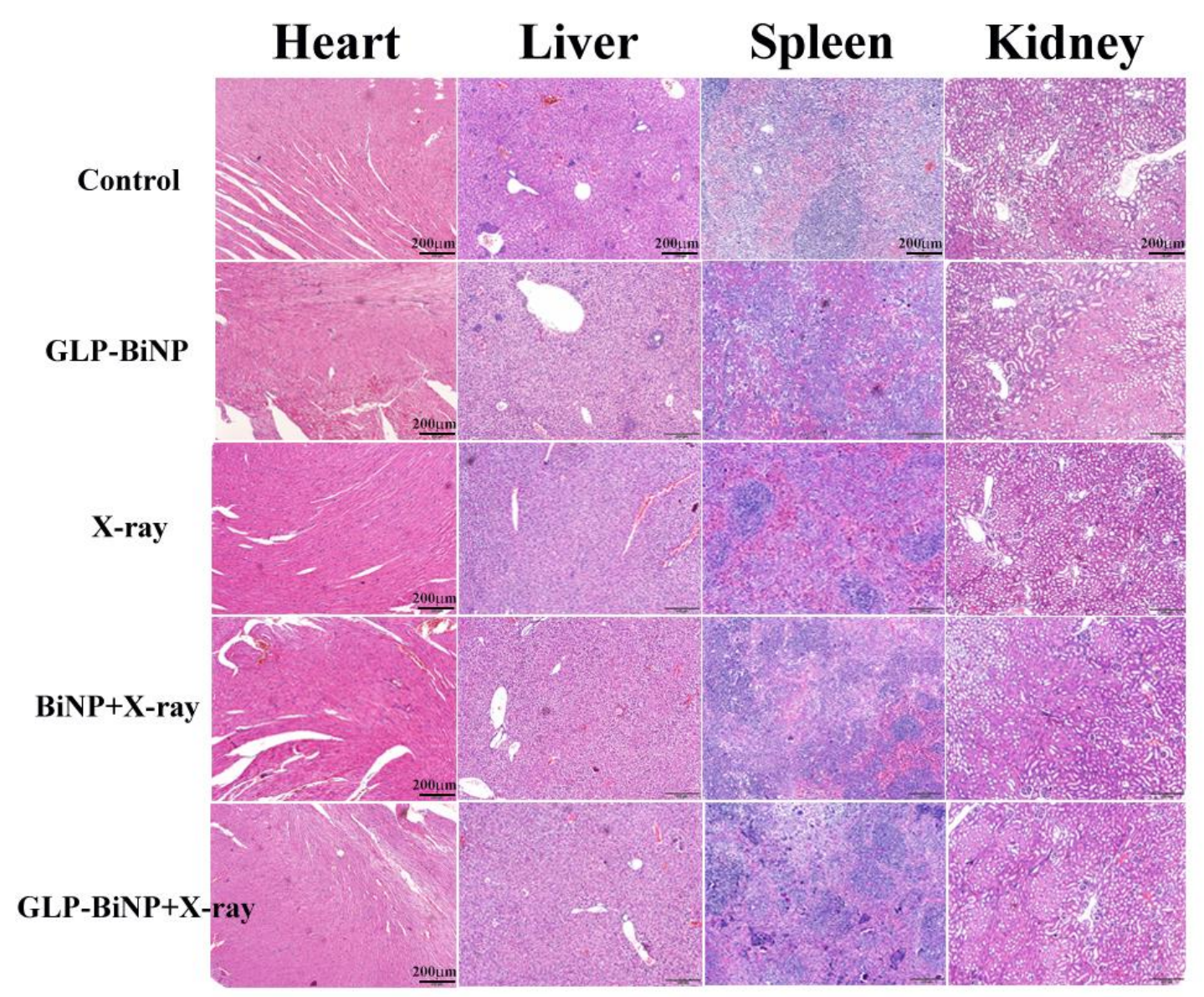

Figure S12. Pathological observations of tissues collected from mice bearing 4T1 tumors.

Tumor bearing mice were subjected to different treatment for 14 days and heart, liver, spleen and kidney were collected for H\&E staining. 

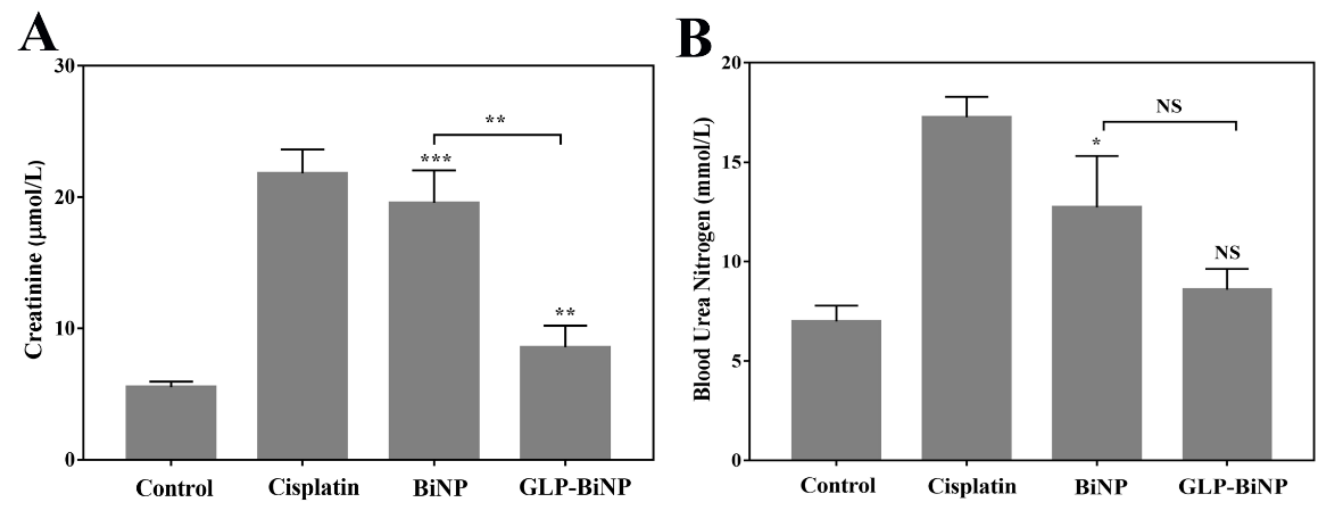

Figure S13. Increased serum creatinine and BUN level by BiNP that can be attenuated by

GLP doping. Mice were injected with PBS, $5 \mathrm{mg} / \mathrm{kg}$ GLP-BiNP or BiNP by i.v. route, or 17 $\mathrm{mg} / \mathrm{kg}$ cisplatin by intraperitoneal injection for three days. (A) Serum creatinine and (B) Blood urea nitrogen (BUN). This specific lot of BiNP showed kidney injury marked as the increased level of creatinine and BUN, while GLP doping significantly reduced kidney injury. All data represent mean \pm S.D. (n=3). NS: Non-significant, $* P<0.05, * * P<0.01, * * * P<0.001$ compared to control unless specified. 


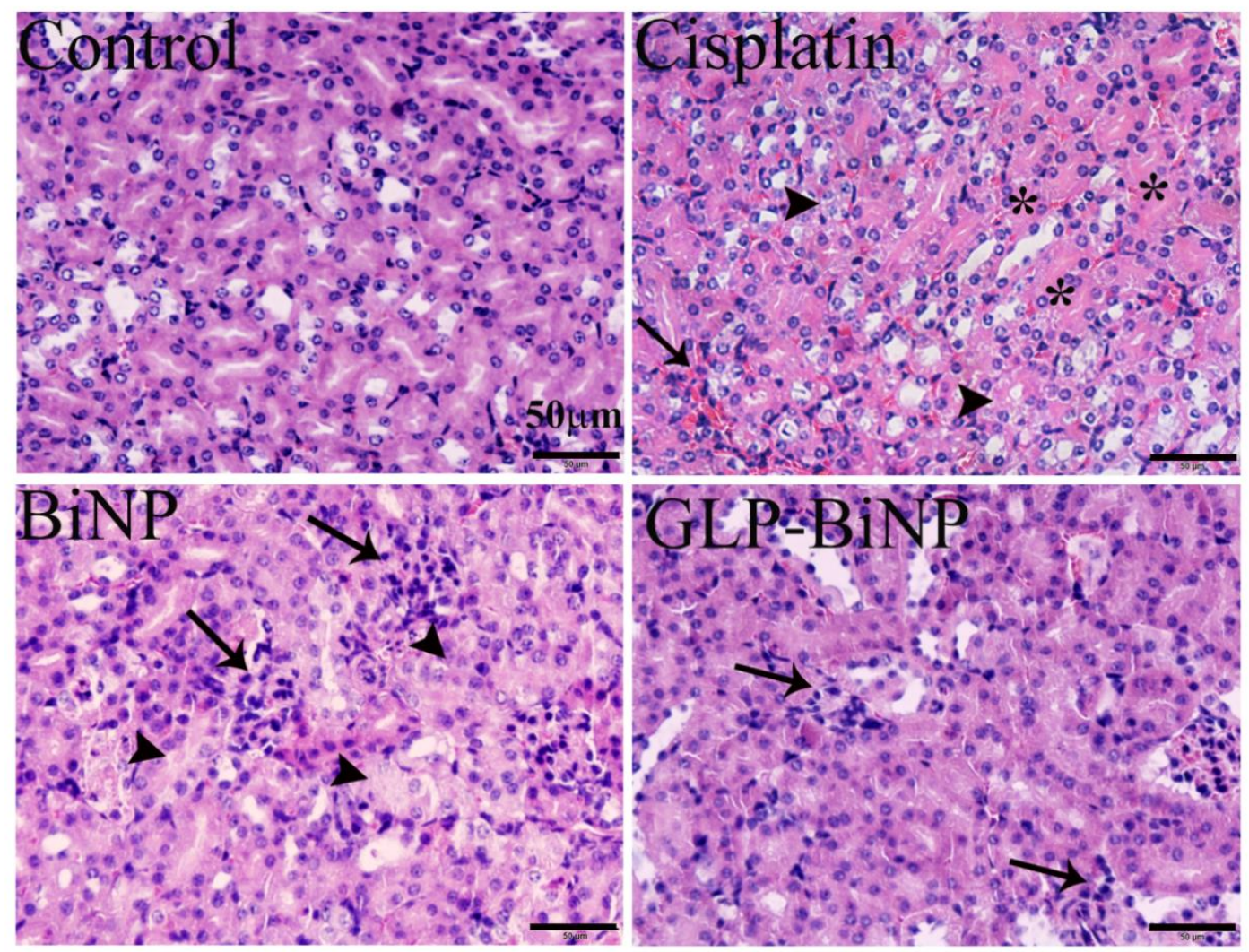

Figure S14. Pathological alteration of kidney by BiNP that can be alleviated by GLP doping.

Intact proximal tubules are found in control group. Renal tubule necrosis and cast (asterisk) can be frequently observed in cisplatin treated groups. BiNP showed vacuolized cytoplasm (arrow head), while GLP-BiNP treated group only showed focal and minor inflammatory infiltration (arrow). The scale bar represents $50 \mu \mathrm{m}$. 